\title{
The incidence of salivary carcinoma ex pleomorphic adenoma: A SEER database review and review of the literature
}

\author{
Carl Rebhun, Richard O. Wein
}

1. School of Medicine, Tufts University, United States. 2. Department of Otolaryngology-Head and Neck Surgery, Tufts Medical Center, United States.

Correspondence: Carl Rebhun. Address: Tufts University School of Medicine, 145 Harrison Avenue, Boston, MA 02111, United States. Email: carl.rebhun@tufts.edu

Received: December 30, 2014

Accepted: January 25, 2015

Online Published: February 11, 2015

DOI : $10.5430 /$ jst.v5n1p26

URL: http://dx.doi.org/10.5430/jst.v5n1p26

\section{Abstract}

Our review included 988 cases of carcinoma ex pleomorphic adenoma (CXPA) in the Surveillance, Epidemiology, and End Results database from 1973-2011 and includes the parotid, submandibular gland, sublingual gland, oral cavity and pharynx. The most common location for CXPA was the parotid gland with a decrease in the reported overall incidence rate noted since 1973. A racial predilection was noted (Black greater than White) and a higher incidence was noted in males. Grade III CXPA had the worst 5- and 10-year disease specific survival. The overall average incidence rate of CXPA for 1973-2011 was 0.68 persons per million. The overall 5- and 10-year survival for CXPA was $63.2 \%$ and $44.6 \%$, respectively.

\section{Key words}

Carcinoma ex pleomorphic adenoma, Carcinoma ex mixed tumor, Carcinoma ex benign adenoma, Salivary gland malignancy, Epidemiology, SEER database

\section{I ntroduction}

Carcinoma Ex Pleomorphic Adenoma (CXPA) is an extremely rare malignant neoplasm that is defined as a pleomorphic adenoma (PA), or mixed tumor, from which an epithelial malignancy has derived ${ }^{[1]}$. Whether CXPA is a de novo malignancy or a malignant transformation from a benign PA is still up to debate ${ }^{[2]}$. CXPA is also known as carcinoma ex mixed tumor, carcinoma ex adenoma, carcinoma ex benign pleomorphic adenoma, and pleomorphic ex carcinoma ${ }^{[3]}$. Classification of CXPA is difficult for multiple reasons. First, as already mentioned, CXPA is a rare entity, and literature on the subject is sparse, so physicians may not have the information necessary to accurately diagnose the condition. Second, some PAs are histologically malignant but behave in a benign manner ${ }^{[4]}$, while other PAs are histologically benign but metastasize ${ }^{[5]}$. Lastly, diagnosis is complicated because the malignant component is often small and overlooked ${ }^{[6,7]}$. Most commonly in the major salivary glands, CXPA has also been diagnosed in the minor salivary glands and other locations in the oral cavity and pharynx ${ }^{[2,8-10]}$. 
Due to its rare nature, the epidemiologic characteristics have been difficult to define. Published series typically reflect the experience of a limited number of patients from a single facility: Gerughty - $25^{[7]}$, Mariano - $38^{[2]}$, Nouraei - $28^{[11]}$, Lewis - $73^{[6]}$, Zbaren - $24^{[12]}$, Szwedowicz - $12^{[13]}$, Katabi - $43^{[14]}$, and Zhao - $51^{[15]}$. The largest reported series of CXPA analyzed included 278 patients. This study was a SEER database review but focused on prognostic factors for survival and was limited to CXPA in the parotid gland from 1988-2009. Our review included 988 cases of CXPA in the SEER database from 1973-2011 and includes the parotid, submandibular gland, sublingual gland, oral cavity and pharynx. To our knowledge, this was the largest retrospective cohort study in the literature at the time of publication. The purpose of the study was to compare the epidemiologic features, including anatomic location, racial and sexual predilection, incidence rate, and survival of CXPA with previous conclusions.

\section{Methods}

The November, 2013 submission of the Surveillance, Epidemiology, and End Results (SEER) database was searched for patients with a diagnosis of CXPA using the International Classification of Diseases for Oncology, 3rd Edition (ICD-O-3) histology codes 8022/3 (pleomorphic carcinoma), 8940/3 (mixed tumor, malignant, NOS), and 8941/3 (carcinoma in pleomorphic adenoma). The cases were further specified ICD-O-3 site recode for oral cavity and pharynx. Two sets of data in the SEER database, each containing information from different registries and years between 1973 and 2011, were used for different purposes. Incidence data were provided by the SEER 9 Registries Research Database, Nov 2013 submission (1973-2011), which covers approximately 9.4\% of the US population and includes the following registries: San Francisco-Oakland SMSA, Connecticut, Detroit (Metropolitan), Hawaii, Iowa, New Mexico, Seattle (Puget Sound), Utah, and Atlanta (Metropolitan). Frequency and survival data were provided by the SEER 18 Registries Research Database, Nov 2013 submission (1973-2011), which covers approximately 27.8\% of the US population and includes the same registries at the SEER 9 plus the following registries: San Jose-Monterey, Los Angeles, Alaska Natives, Rural Georgia, California excluding SF/SJM/LA, Kentucky, Louisiana, New Jersey, and Greater Georgia. All data was collected and analyzed using the SEER*Stat 8.1.5 software (National Cancer Institute, Bethesda, MD).

Epidemiologic characteristics analyzed included age at diagnosis, sex, race, histologic grade, and primary location. Race was divided into white, black, and other (American Indian/Alaska Native, Asian/Pacific Islander, or unknown). Incidence and annual percentage change (APC) of CXPA were stratified according to sex and race. Google Sheets (Google, Mountain View, CA) was used to graph data. Both 5- and 10- year disease specific survival (DSS) were calculated for overall CXPA and CXPA stratified by histologic grade. Survival was estimated using Kaplan-Meier analysis on the SEER*Stat 8.1.5 software and was graphed using Microsoft Office Excel 2011 (Microsoft Corporation, Redmond, WA). A probability value ( $p$-value) of $<0.05$, obtained using the statistical software SEER*Stat 8.1.5, was considered statistically significant for all tests. International review board approval was not required as information on the SEER database is publicly available and does not expose personal patient information.

\section{Results}

\subsection{Frequency data}

There were a total of 988 cases of CXPA in the oral cavity and pharynx reported in the SEER database from 1973-2011, covering $27.8 \%$ of the US population. Patient characteristics are shown in Table 1 . CXPA was more common in males than in females (53.24\% vs. $46.76 \%$, respectively). Of the cases, White patients represented $83.50 \%$ of the patient pool, Black patients $10.53 \%$, and other races 5.67\%. There was a mean age of diagnosis of 61.6 years. Only 419 cases in the database reported grading information. Of those, most were grade III (poorly differentiated; 43.9\%), grade IV (undifferentiated; 23.6\%) and grade II (moderately differentiated; 22.7\%) were second and third most, respectively, and grade I (well differentiated; 9.8\%) was the rarest. Locations varied in many places throughout the head and neck. The 
parotid gland (66.30\%), submandibular gland (14.98\%), and major salivary gland NOS (7.39\%) were the most common locations. Of the minor salivary glands, locations in the hard palate $(2.23 \%)$ and soft palate $(1.42 \%)$ were the most common.

Table 1. Cohort Characteristics of Carcinoma ex Pleomorphic Adenoma from SEER 18 Database 1973-2011

\begin{tabular}{|c|c|c|}
\hline \multirow{2}{*}{ Item } & $\mathbf{n}$ & Percent \\
\hline & 988 & $100 \%$ \\
\hline \multicolumn{3}{|l|}{ Gender } \\
\hline Male & 526 & $53.24 \%$ \\
\hline Female & 462 & $46.76 \%$ \\
\hline \multicolumn{3}{|l|}{ Race } \\
\hline White & 825 & $83.50 \%$ \\
\hline Black & 104 & $10.53 \%$ \\
\hline Other & 56 & $5.67 \%$ \\
\hline Unknown & 3 & $0.30 \%$ \\
\hline \multicolumn{3}{|l|}{ Age at diagnosis } \\
\hline $10-14$ years & 1 & $0.10 \%$ \\
\hline $15-19$ years & 4 & $0.40 \%$ \\
\hline 20-24 years & 5 & $0.51 \%$ \\
\hline $25-29$ years & 15 & $1.52 \%$ \\
\hline 30-34 years & 26 & $2.63 \%$ \\
\hline 35-39 years & 42 & $4.25 \%$ \\
\hline 40-44 years & 55 & $5.57 \%$ \\
\hline $45-49$ years & 75 & $7.59 \%$ \\
\hline 50-54 years & 100 & $10.12 \%$ \\
\hline $55-59$ years & 114 & $11.54 \%$ \\
\hline $60-64$ years & 110 & $11.13 \%$ \\
\hline $65-69$ years & 124 & $12.55 \%$ \\
\hline $70-74$ years & 88 & $8.91 \%$ \\
\hline 75-79 years & 102 & $10.32 \%$ \\
\hline $80-84$ years & 66 & $6.68 \%$ \\
\hline $85+$ years & 61 & $6.17 \%$ \\
\hline Age at Diagnosis, mean & 61.6 years & \\
\hline \multicolumn{3}{|l|}{ Grade } \\
\hline I (Well differentiated) & 41 & $4.15 \%$ \\
\hline II (Moderately Differentiated & 95 & $9.62 \%$ \\
\hline III (Poorly Differentiated) & 184 & $18.62 \%$ \\
\hline IV (Undifferentiated; anaplastic) & 99 & $10.02 \%$ \\
\hline Unknown & 569 & $57.59 \%$ \\
\hline \multicolumn{3}{|l|}{ Primary Location } \\
\hline Mouth, NOS* & 31 & $3.11 \%$ \\
\hline Hard Palate & 22 & $2.23 \%$ \\
\hline Soft Palate & 14 & $1.42 \%$ \\
\hline Palate, NOS* & 11 & $1.11 \%$ \\
\hline Cheek Mucosa & 11 & $1.11 \%$ \\
\hline Parotid Gland & 655 & $66.30 \%$ \\
\hline Submandibular Gland & 148 & $14.98 \%$ \\
\hline Sublingual Gland & 2 & $0.20 \%$ \\
\hline Major Salivary Gland, NOS* & 73 & $7.39 \%$ \\
\hline Pharynx, NOS* & 21 & $2.11 \%$ \\
\hline
\end{tabular}

* NOS: Not Otherwise Specified 


\subsection{I ncidence data}

The overall APC, or incidence trend, of CXPA from 1973-2011 was $-1.18 \%$ ( $p<.01$, CI -1.91 to -0.45 ). The plotted annual incidences can be seen in Figure 1. Incidence rates were based off of a total of 607 cases of CXPA documented in the 9 SEER registries from 1973-2011, representing 9.4\% of the US population. The overall average incidence rate of CXPA for 1973-2011 was 0.68 persons per million. The incidence rate for the White population was 0.71 persons per million, for the Black population was 0.77 , and for the other races was 0.34 . For males the incidence rate was 0.80 persons per million and for females 0.59 . The CXPA incidence rates for certain age groups for male, female, and overall population can be seen in Figure 2.

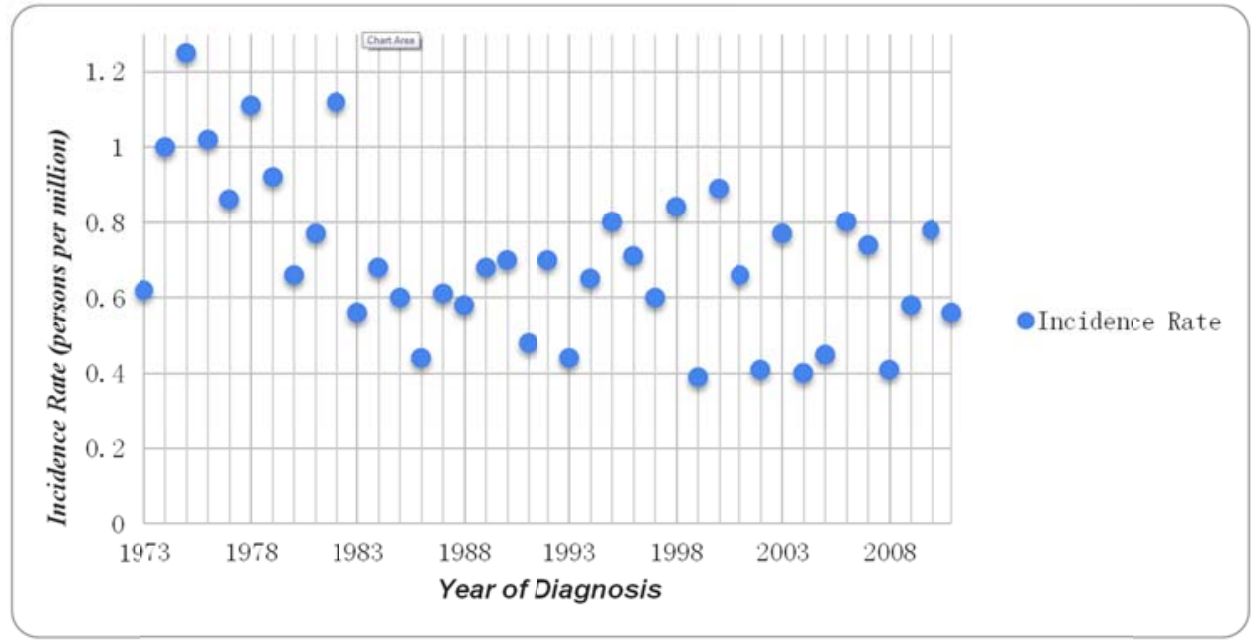

Figure 1. Overall Incidence Trend of Carcinoma Ex Pleomorphic Adenoma from 1973-2011

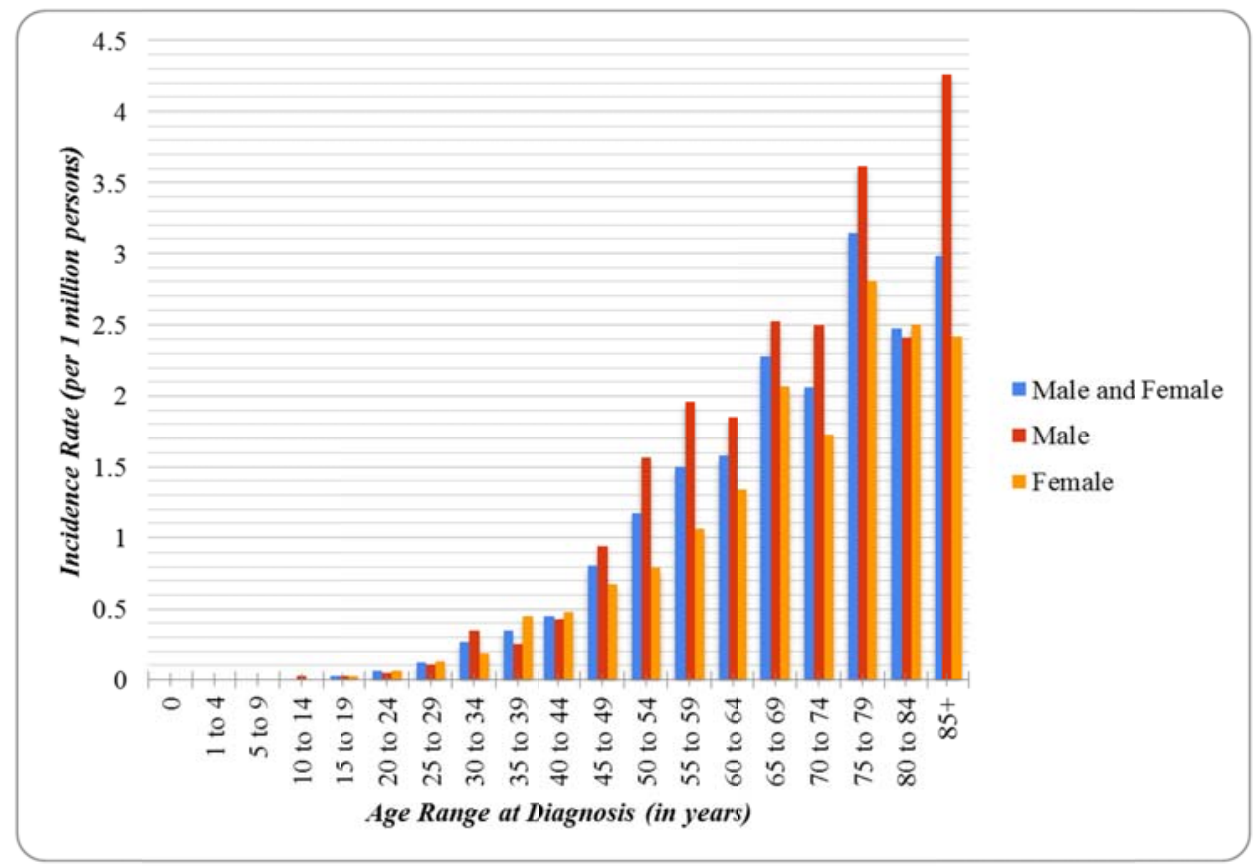

Figure 2. Carcinoma Ex Pleomorphic Adenoma Incidence Rates Stratified by Sex and Age of Diagnosis for SEER 9 Registries, 1973-2011 


\subsection{Survival data}

Both 5- and 10-year overall and DSS were calculated for CXPA. There were a total of 868 cases in the SEER database from 18 registries from 1973-2011. The overall 5- and 10-year survival for CXPA was 63.2\% and 44.6\%, respectively. DSS for 5- and 10-year survival was 73.7\% and 66.3\%, respectively. In addition to the CXPA in general, 5- and 10-year DSS was calculated for CXPA grades I-IV, as shown in Figure 3. Survival decreases as the grade increases except for grades III and IV. Five year DSS for CXPA grade III is $57.0 \%$ and for grade IV is $67.4 \%$. For comparison, grade I and II have 5 year DSS of $95.9 \%$ and $89.7 \%$, respectively.

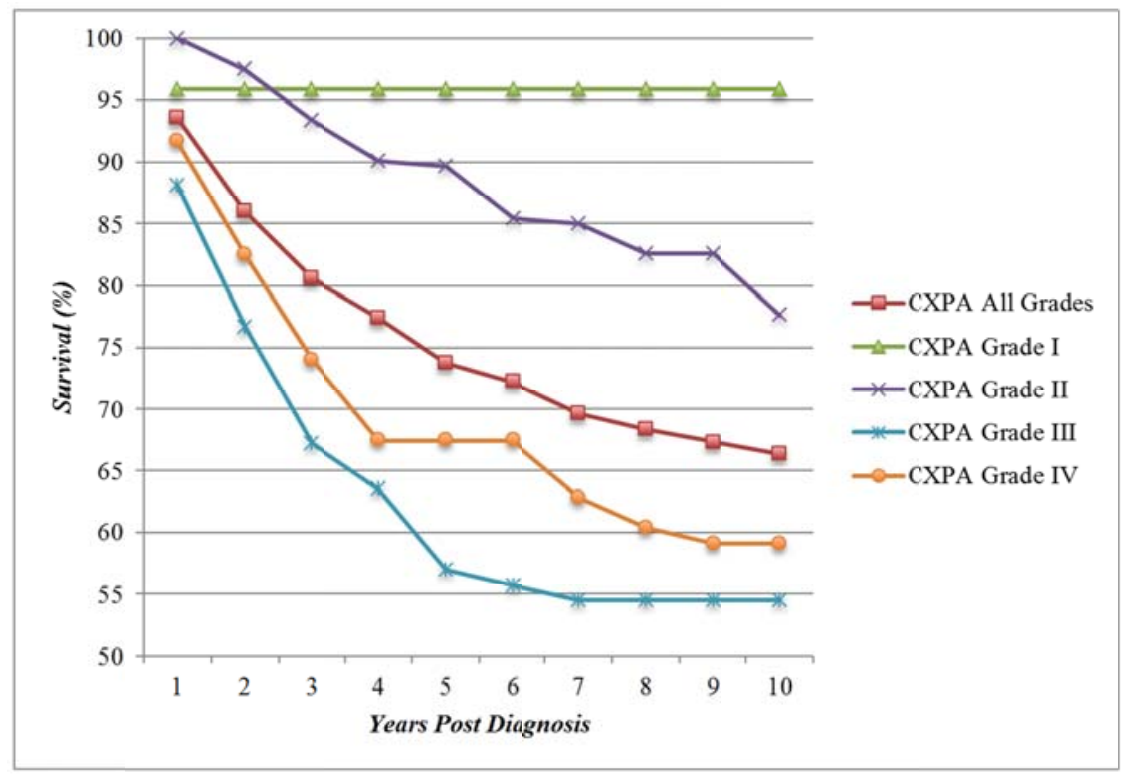

Figure 3. Ten Year Disease Specific Survival of Carcinoma Ex Pleomorphic Adenoma Stratified by Histologic Grade

\section{Discussion}

To our knowledge, this study is the largest population-based epidemiologic study of CXPA in the literature. Previous studies have focused on relatively small-scale cohorts derived from single institutions. The SEER database analysis allowed for the confirmation and contradiction of previously accepted epidemiologic findings of CXPA. Frequency analysis of CXPA from 1973-2011 resulted in 988 total reported cases and an average annual incidence rate of 0.68 persons per million. The previously widely accepted incidence was 0.17 persons per million ${ }^{[10]}$. Gnepp utilized cases in the SEER database from 1973-1977 to arrive at his incidence results. These results are now outdated and, as our analysis shows, the overall annual incidence is about four times higher, albeit still extremely rare with less than 1 person per million. The incidence trend of CXPA has not been previously shown. Here, we provide a graph of yearly incidences from 1973-2011, showing a downward trend and a statistically significant decrease in annual percentage change of CXPA. Reasons for this downward trend are unknown, but one can hypothesize that more patients are having surgical resection before malignant transformation can occur. The incidence of PA has been reported as ranging from 3.05 persons per one hundred thousand, making it forty times more common than CXPA $^{[16]}$.

CXPA is slightly more common in males than in females with a male to female ratio of 1.1:1. The male to female ratio has been varied in the literature. Our findings were closest to two studies' results that showed the male to female ratio was approximately even ${ }^{[2,9]}$. This finding differed from other reports that found males were significantly more likely to be diagnosed with CXPA. Previous reports ${ }^{[6,11,15]}$ showing a male preponderance had male to female ratios ranging from 1.8-2.4:1. Other reports ${ }^{[10,13,14]}$, showing a higher female likelihood of developing CXPA, had male to female ratios of 
1:1.2-1.7. In addition to frequency data, we determined average annual incidence rates for sex. In each age group, males had a higher incidence than females, with an average incidence of 0.8 and 0.59 persons per million, respectively. It is unknown what may predispose males to this higher incidence.

CXPA and race preponderance has not been mentioned in previous studies. Our findings that white patients represent $83.50 \%$ of the patient pool is similar to the findings of Chen et al. whose results showed $85.6 \%$ of patients were white ${ }^{[17]}$. Our results also show that black patients represented $10.53 \%$ and other races represented $5.67 \%$. This frequency data is perhaps less useful than the average incidence data that adjusts for population size according to race from 1973-2011. Incidence data shows a different story, with the black population having a slightly higher incidence of CXPA than the white population and other races. Respectively, the incidence rates are $0.77,0.71$, and 0.34 persons per million, contrasting a previous epidemiological study that concluded there was no racial predilection ${ }^{[10]}$.

Average age at diagnosis is relatively constant throughout the literature reporting that most patients are diagnosed in the 7 th decade of life ${ }^{[3,6,10-12,14]}$. Our analysis of the SEER database showed an average age of diagnosis of 61.6 years. Figure 2 shows that average annual incidence increases as age increases in both males and females. This may indicate that age is an independent risk factor for developing CXPA, however further analysis is needed. Studies support this notion reporting that increased preoperative duration of PA increases the risk of transformation to $\mathrm{CXPA}^{[3,10]}$. Others show that the average age for PA is significantly lower than that for CXPA ${ }^{[13]}$.

CXPA has been reported to develop in various locations in the head and neck. The most common primary location for CXPA is in the major salivary glands, where a total of $88.87 \%$ of CXPA tumors arose. Our results show the vast majority of these occurring in the parotid gland followed by the submandibular gland, with only two cases reported in the sublingual gland. These results are consistent with previous reports ${ }^{[1,2,10,15]}$. Less commonly reported locations include the minor salivary glands, most notably in the palate. The SEER database provided figures of $2.23 \%$ in the hard palate, and $1.42 \%$ and $1.11 \%$ in the soft palate and palate NOS, respectively. This total of $4.76 \%$ supports other reports ${ }^{[2,9,10,14]}$. Location is important because a previous SEER database report analyzing survival data focused solely on the parotid gland, and therefore excluded a significant amount of CXPA cases ${ }^{[17]}$.

We determined overall CXPA disease specific survival (DSS) and stratified the DSS according to histologic grading of the tumor. For all cases of CXPA, the 5- and 10-year DSS were 73.7\% and 66.30\%, respectively. Previous reports of survival have varied dramatically, but most reported shorter survival than reported here, with 5 year DSS of $37 \%{ }^{[6]}, 44 \%{ }^{[11]}$, $53.5 \%{ }^{[15]}, 75 \%{ }^{[12]}$, and $80.8 \%{ }^{[17]}$. With the largest published cohort of CXPA, our results show that survival of CXPA is not as poor as previously believed ${ }^{[17]}$.

With regard to histologic grading of CXPA, previous studies were not specific in their determination, reporting only that $89 \%$ were grade III or IV and that $9 \%$ were grade I or II ${ }^{[6]}$. Of the 419 cases in the SEER database that reported grading information, most were grade III, followed by frequencies of grades IV and II and grade I was least frequent. We found that grading information had an effect on the survival outcome. Others have noted that high-grade neoplasms have significant effects on survival ${ }^{[6,15]}$. However, our results show that grade III neoplasms have the worst survival, worse even than grade IV. The greater effectiveness of radiotherapy on higher-grade tumors could contribute to this outcome, however, it is unclear whether or not radiotherapy has a survival advantage. Nonetheless, radiation treatment should be offered post-operatively for those patients with factors for a poor prognosis ${ }^{[15]}$.

There are some limitations of this study. Only cases reported to the SEER database could be analyzed. Therefore, we were dependent on others' work to correctly identify and submit cases of CXPA. Because there is debate on how to characterize CXPA, some cases in the SEER database may be incorrectly classified as CXPA, while other cases may have been incorrectly classified as not CXPA. Selection bias may have played a factor as this is a retrospective cohort study. 


\section{Conclusion}

In summary, CXPA was most commonly seen in the parotid gland. There was a racial and sexual predilection toward Blacks and males, respectively, and there has been a decline in the overall incidence of CXPA between 1973 and 2011. Grade III CXPA was associated with the worst disease specific survival, while the overall 5- and 10-year survival was $63.2 \%$ and $44.6 \%$, respectively, which was better than previously thought. Future research should focus on better characterizing CXPA in terms of its pathological features, as this will help solidify future epidemiologic studies.

\section{Competing interests}

The authors declare that they have no competing interests.

\section{References}

[1] Di Palma S. Carcinoma ex pleomorphic adenoma, with particular emphasis on early lesions. Head Neck Pathol. 2013 Jul; 7 Suppl 1: S68-76. http://dx.doi.org/10.1007/s12105-013-0454-z

[2] Mariano FV, Noronha AL, Gondak RO, et al. Carcinoma ex pleomorphic adenoma in a Brazilian population: clinic-pathological analysis of 38 cases. Int J Oral Maxillofac Surg. 2013 Jun; 42(6): 685-92. http://dx.doi.org/10.1016/j.ijom.2013.02.012

[3] Antony J, Gopalan V, Smith RA, et al. Carcinoma ex pleomorphic adenoma: a comprehensive review of clinical, pathological and molecular data. Head and Neck Pathol. 2012; 6: 1-9. PMid:21744105 http://dx.doi.org/10.1007/s12105-011-0281-z

[4] Di Palma S, Skalova A, Vanleek T, et al. Non-invasive (intracapsular) carcinoma ex pleomorphic adenoma: recognition of focal carcinoma by HER-2/neu and MIB1 immunohistochemistry. Histopathology. 2005 Feb; 46(2): 144-52. http://dx.doi.org/10.1111/j.1365-2559.2005.02058.x

[5] Wenig BM, Hitchcock CL, Ellis GL, et al. Metastasizing mixed tumor of salivary glands. A clinicopathologic and flow cytometric analysis. Am J Surg Pathol. 1992 Sep; 16(9): 845-58. http://dx.doi.org /10.1097/00000478-199209000-00003

[6] Lewis JE, Olson KD, Sebo TJ. Carcinoma ex pleomorphic adenoma: pathologic analysis of 73 cases. Hum Pathol. 2001 Jun; 32(6): 596-604. http://dx.doi.org/10.1053/hupa.2001.25000

[7] Gerughty RM, Scofield HH, Brown FM, et al. Malignant mixed tumors of salivary origin. Cancer. 1969 Sep; $24(3)$ : $471-86$. http://dx.doi.org/10.1002/1097-0142(196909)24:3<471::AIDCNCR2820240309>3 .0.CO;2-0

[8] Waldron CA, el-Mofty SK, Gnepp DR. Tumors of the intraoral minor salivary glands: a demographic and histologic study of 426 cases. Oral Surg Oral Med Oral Pathol. 1988 Sep; 66(3): 323-33. http://dx.doi.org/10. 1016/0030-4220(88)90240-X

[9] Weiler C, Zengel P, van der Wal JE, et al. Carcinoma ex pleomorphic adenoma with special reference to the prognostic significance of histological progression: a clinicopathological investigation of 41 cases. Histopathology. 2011 Oct; 59(4): $741-50$. http://dx.doi.org/10.1111/j.1365-2559.2011.03937.x

[10] Gnepp DR. Malignant mixed tumors of the salivary glands: a review. Pathol Annu. 1993; 28 Pt 1: 279-328. PMid:8380049

[11] Nouraei SA, Hope KL, Kelly CG, et al. Carcinoma ex benign pleomorphic adenoma of the parotid gland. Plast Reconstr Surg. 2005 Oct; 116(5): 1206-13. http://dx.doi.org/10.1097/01.prs.00001816 54.68120.0f

[12] Zbaren P, Zbaren S, Caversaccio, et al. Carcinoma ex pleomorphic adenoma: diagnostic difficulty and outcome. Otolaryngol Head Neck Surg. 2008 May; 138(5): 601-5. http://dx.doi.org/10.1016/j .otohns.2008.01.013

[13] Szwedowivz P, Osuch-Wójcikiewicz E, Bruzgielewicz A, et al. Malignant mixed tumors of the parotid gland: a review of the Department of Otolaryngology Medical University of Warsaw experience. Otolaryngol Pol. 2012 Sep; 66(4 Suppl): 4-10.

[14] Katabi N, Gomez D, Klimstar DS, et al. Pathology factors of recurrence in salivary carcinoma ex pleomorphic adenoma, with emphasis on the carcinoma histologic subtype: a clinicopathologic study of 43 cases. Hum Pathol. 2010 Jul; 41(7): 927-34. http://dx.doi.org/10.1016/j.humpath.2009.12.011

[15] Zhao J, Wang J, Yu C, et al. Prognostic factors affecting the clinical outcome of carcinoma ex pleomorphic adenoma in the major salivary gland. World J Surg Oncol. 2013 Aug 8; 11(1): 180. http://dx.doi.org/10.1186/ 1477-7819-11-180

[16] Pinkston JA, Cole P. Incidence of salivary gland tumors: results from a population-based study. Otolaryngol Head Neck Surg. 1999 Jun; 120(6): 834-0. http://dx.doi.org/10.1016/S0194-5998(99)70323-2

[17] Chen MM, Roman SA, Sosa JA, et al. Predictors of survival in carcinoma ex pleomorphic adenoma. Head Neck. 2014 Sep; 36(9): 1324-8. PMid:23956034. 